\title{
A study of intestinal parasitic infections in HIV infected individuals in Puducherry
}

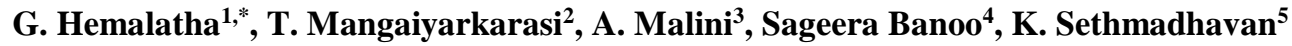 \\ ${ }^{1}$ Assistant Professor, ${ }^{2-4}$ Associate Professor, ${ }^{5}$ Professor, Dept. of Microbiology, ${ }^{1,5}$ Aarupadaiveedu Medical College and Hospital, \\ Vinayaka Missions University, Puducherry, ${ }^{2}$ Sri Manakula Vinayagar Medical College\& Hospital, Pondicherry University, \\ Puducherry, ${ }^{3}$ Indira Gandhi Medical College, Pondicherry University, Puducherry, ${ }^{4}$ Dhanalakshmi Medical College \& Hospital,
} The Tamilnadu Dr MGR Medical University, Tamilnadu, India

*Corresponding Author:

Email: drghl2010@gmail.com

\begin{abstract}
Introduction: Human immuno deficiency virus infection has become a pandemic. Infection with HIV results in a chronic illness characterized by a progressive decline in cell-mediated immune function. Diarrhoea occurs in almost $90 \%$ of patients with HIV in developing countries at sometime during the clinical course, and is the presenting symptom of approximately one third of patients with HIV infection.

Aims:

1. To find out the common enteric parasitic pathogens in the HIV infected individuals.

2. To correlate the immune status to the parasitic infection.

3. To find out the symptomatic correlation to the pathogens.

Settings and Design: The cross sectional study was carried out in Puducherry for a period of 15 months.

Materials and Methods: Two consecutive stool samples were collected from from HIV seropositive patients. The samples were concentrated and subjected to stool concentration, iodine and saline mounts \& Kinyoun's staining.

Statistical analysis used: Epi info 6 computer software package [CDC, Atlanta, Georgia, USA, 1995].

Results: Of the 22 diarrhoeic stools of HIV cases, 7(31.8\%) yielded parasites on Kinyoun's staining; all 7(100\%) were opportunistic protozoan pathogens. The prevalence of intestinal parasites among the HIV infected patients is as follows: Cryptosporidium sp. and Microsporidium sp., each were found in 6.3\% of HIV seropositive cases. Strongyloides was seen in $2.5 \%$ of 80 seropositive cases. The mean $\mathrm{CD}_{4}$ counts in cases with parasites are listed as follows: Cryptosporidium sp.-133.2 cells, Microsporidium sp.-896 cells, Strongyloides stercoralis-228.5, Entamoeba histolytica/dispar-522 cells, Giardia sp. - 884 cells. Cryptosporidium sp. and Strongyloides stercoralis are found more in HIV seropositive cases with $\mathrm{CD}_{4}$ counts less than 200 cells; $(\mathrm{p}=0.002, \mathrm{OR}=36,95 \% \mathrm{CI}=4-56)$ for Cryptosporidium sp. and ( $\mathrm{OR}=14.6,95 \% \mathrm{CI}=1.5-39.6)$ for Strongyloides stercoralis. There is a significant correlation between $\mathrm{CD}_{4}$ counts $<200$ and diarrhoea presentation, $(\mathrm{p}=0.005)$; increased risk, $\mathrm{OR}=16.76(95 \%$ CI 1.63-106.59).
\end{abstract}

Conclusions: The present study shows that Cryptosporidium is the predominant enteric protozoan parasite.

Keywords: HIV, Diarrhoea, Cryptosporidium, Kinyoun staining, $\mathrm{CD}_{4}$ counts

Key Messages: Enteric parasitic pathogens (opportunistic) in HIV infected individuals is common in patients with normal $\mathrm{CD}_{4}$ counts and antibiotic prophylaxis.

\section{Introduction}

Human immuno deficiency virus infection has become a pandemic. No country is spared from this viral infection. In 2016, an estimated 36.7 million people were living with HIV (including 1.8 million children) - with a global HIV prevalence of $0.8 \%$ among adults. ${ }^{1}$ In 2016, HIV prevalence in India was an estimated $0.3 \% .^{2}$ This figure is small compared to most other middle-income countries but because of India's huge population (1.324 billion) this equates to 2.1 million people living with HIV. ${ }^{2}$ Infection with HIV results in a chronic illness characterized by a progressive decline in cell-mediated immune function. Because of the profound immunosuppression, HIV infected patients become highly susceptible to a wide range of common as well as opportunistic infections. The gastrointestinal tract is the major target organ for opportunistic infections. Diarrhoea is the common clinical manifestation in these infections. Reports indicate that diarrhoea occurs in 30-60\% of AIDS patients in developed countries and in about $90 \%$ of
AIDS patients in developing countries. In Africa, this disorder has been associated with "Slim disease", a term that describes the great loss of weight that accompanies diarrhoea.

Enteric parasites are one of the most common infections among HIV/AIDS patients. ${ }^{3}$ Studies report that several factors including lower CD4 T-cell count, diarrhea, living in a rural area and poor nutrition have been associated with the higher prevalence of enteric parasitic infection in HIV-infected individuals. ${ }^{4}$

Chronic diarrhoea is a common problem for patients with HIV, especially those with the advanced disease. Chronic diarrhoea significantly reduces the quality of life in patients with HIV infection and is an independent marker of poor prognosis and also an independent predictor of mortality in patients with AIDS. ${ }^{5}$ Although, diarrhoea can occur both in early and advanced HIV infection, its persistence for more than one month in association with unexplained weight loss of at least $10 \%$ is an AIDS defining condition. ${ }^{6}$ 
Common enteric parasites causing diarrhoea in HIV infected patients are Cryptosporidium sp., Microsporidia,Isospora belli, Cyclospora cayetanensis, Giardia lamblia, Entamoeba histolyticaldispar, Blastocystis hominis, Strongyloides stercoralis.

Intestinal protozoan parasites have gained importance as opportunistic pathogens in HIV infected patients. These parasites are associated with persistent voluminous diarrhoea in AIDS patients.

Diarrhoea associated with these infections have significantly affected the quality of life leading to an increase in morbidity and mortality in HIV patients. Since HIV infection is spreading with rapidity and diarrhoeal illness due to parasitic etiology is increasing among HIV infected subjects, clinical microbiologists ought to be aware of the wide spectrum of these intestinal protozoan infections. This prompted us to undertake the present study to know the prevalence of intestinal parasites in HIV seropositive patients.

T-lymphocytes which are responsible for cell mediated immunity are important for defence against fungi, protozoa, intracellular bacterial infections, mycobacteria and viruses.

T-cell immunity is mediated either by the helper/inducer or $\mathrm{CD}_{4}+\mathrm{T}$-cells and the cytotoxic/immunoregulatory or $\mathrm{CD}_{8}+\mathrm{T}$-cells. Helper/inducer cells are defined phenotypically by the presence on its surface of the $\mathrm{CD}_{4}$ molecule, which serve as the primary cellular response for HIV.

Infection with HIV irrespective of its type, subtype and route of infection leads to protracted disease and depletion of $\mathrm{CD}_{4}$ cells in most cases resulting in AIDS.

\section{Materials and Methods}

The present study comprises of identification of enteric parasites in HIV seropositive individuals. The study was carried out in Puducherry for a period of 15 months. A total of 80 patients who were HIV seropositive were included in the study. A control group of 20 non-HIV subjects was also included in the study. Informed consent was taken from participants.

Institutional ethics committee clearance was taken.

The HIV status of the cases was noted from the records. Detailed clinical history of patients was obtained regarding the duration of seropositive status, ART treatment, TB treatment, complaints of diarrhoea, any antibiotics treatment taken for diarrhoea, weight loss, loss of appetite, vomiting and fever. Diarrhoea was defined as two or more liquid or three or more soft stools per day; diarrhoea of duration more than 30 days was considered chronic diarrhoea. Patients already on antibiotic treatment were excluded from the study. Also the history of associated opportunistic infections like Tuberculosis, Vaginal candidiasis and Oral thrush were noted. Recently done $\mathrm{CD}_{4}$ counts, haemoglobin, total cell counts were also documented from records. The individuals not willing to give samples were not included in the study.

\section{Specimen collection and transport}

Stool samples were collected according to WHO standard procedures. Stool specimens were collected in wide mouthed, clean, dry, plastic containers with tight fitting lids. Patients were instructed about the method of stool collection after obtaining an oral informed consent. The patients were asked to collect their stool sample in the morning. Two stool samples on two consecutive days were taken from each patient. They were instructed to avoid contamination of the stool specimen with urine or water. The specimens were brought to the laboratory without undue delay. The specimens were stored in thermocol boxes with ice packs till transport to the laboratory. The specimens after receiving were labelled properly. The specimens were processed, and after processing they were preserved by adding $1-2 \mathrm{gm}$ of stool sample to $8-10 \mathrm{ml}$ of $10 \%$ formalin in the $15-30 \mathrm{ml}$ screw capped vials.

\section{Stool examination}

Macroscopic examination: The specimens were examined by naked eye for colour, consistency, presence of blood, mucus, adult worms or segments of worms.

\section{Microscopic examination:}

1. Saline mount: A loopful of specimen was mixed with a drop of normal saline, placed on a glass slide. A coverslip was placed and the mount was examined under the low and high power objectives of the light microscope.

2. Iodine mount: To the drop of saline emulsion of stool sample, a drop of Lugol's iodine was added and then a coverslip was placed on the preparation. The mount was examined under the low power and high power objectives of the light microscope.

The wet mounts were screened by examining the slides starting from one end of the coverslip. The saline mount was examined for the motile trophozoites, larvae, cysts, ova, puscells, RBCs, bacteria, yeast cells, hyphal elements, fat globules and Charcot Layden crystals. The iodine mount was examined particularly for the study of nuclear characteristics and glycogen mass.

The stool samples were concentrated by Formol ether sedimentation technique. After concentration technique was employed, the saline and iodine mounts were done and examined under the microscope with low and high power objectives. Two mounts in each category were examined for each sample received in the laboratory.

A thin faecal smear is made on a clean glass slide and air dried. Smears were prepared to identify the parasites by Modified Ziehl Neelsen staining technique (Kinyoun's staining).

Method: The air dried smear was fixed in methanol for 2-3 mins. To the fixed smear on the slide rack, strong, cold carbol fuchsin was added and allowed for 10-15 minutes. The slide was rinsed in running tap water and 
$1 \%$ acid-alcohol was added till pink colour on the slide goes off. The slide was rinsed in running tap water. The slide was counter stained with methylene blue for 30 seconds. The slide was rinsed in running tap water. The slide was air dried and examined under oil immersion objective. Two stained smears were examined for each sample received in the laboratory.

Interpretation: Cryptosporidium species oocysts stain bright pink measuring 4-5 $\mu \mathrm{m}$ in diameter and appear as spherules on a pale blue background. Oocysts of cyclospora cayetanensis stain bright pink measuring 8$10 \mathrm{~m}$ in diameter, spherical, on a pale blue background. The degree and proportion of staining varies with individual oocysts. The internal structures take up the stain to varying degrees. Some may appear amorphous, while others may contain the characteristic crescentric form of the sporozoites. Isospora belli oocysts stain pink and appear as large elongated ovoid bodies, measuring $20-33 \mu \mathrm{m} \times 10-19 \mu \mathrm{m}$, tapered at the ends and containing either a granular zygote or two sporoblasts. Yeast cells could also be identified as uniformly stained light pink coloured spherical bodies.

\section{Statistical analysis}

Statistical analysis was performed using the Epi info 6 computer software package [CDC, Atlanta, Georgia, USA, 1995].

The stool samples were macroscopically classified into loose, semisolid and formed stools. For statistical analysis, cases were stratified by age-intervals; CD4 counts $\leq 200,201-400,401-800,>800$ cells; $\mathrm{Hb} \leq$ $10,>10 \mathrm{mg} \%$; WBC counts $\leq 4200,>4200$ cells.

Different characteristics of study participants were described using mean, range and percentage as appropriate. Statistical significance of differences in proportions was evaluated by Pearson's Chi-square test. Fisher Exact test was used in place of Pearson's Chisquare when more than $20 \%$ of cells in contingency tables have expected count less than 5. Diarrhoea as an ordered categorical outcome variable, we used proportional odds model to evaluate the independent effect of HIV and parasitic infection on diarrhoea. Odds ratio was used to measure the strength of association between outcome and its correlates. A given statistical test was reported significant whenever it resulted in a pvalue $<0.05$.

\section{Results}

A total of 80 HIV seropositive patients, control group of 20 HIV seronegative individuals was taken for study as per the inclusion criteria. The control group individuals were randomly selected from those attending out patient department for different complaints.

Out of 80 patients, $47(58.7 \%)$ were males and 33 $(41.3 \%)$ were females. Nearly $28.8 \%$ were belonging to 11-20 years of age group and $27.5 \%$ were from $31-40$ years of age group. Among 46 individuals $>18$ years of age, $35(76.1 \%)$ were married.

Among 80 individuals, $18(22.5 \%)$ were students; $11(13.8 \%)$ were not occupied; $8(10 \%)$ were in the each categories of coolie with mean income of rupees 2750, maid with mean income of rupees 4125 and housewife; $9(11.3 \%)$ were below the age for working and 7(8.8\%) were business persons with mean income of rupees 10700 .

Among 80, 23(28.8\%) were seropositive since past 1 to 2 years; $20(25 \%)$ were seropositive since past 1 year; $21(26.2 \%)$ were seropositive since past 2 to 3 years; $7(8.75 \%)$ in each category were seropositive since past 3 to 4 years and 4 to 5 years; $1(1.25 \%$ ) in each category were seropositive since past 5 to 6 years and more than 6 years period.

Among 80 seropositive patients, 66 (82.5) were on ART treatment.

Among 80, 26(32.5\%) suffered from Tuberculosis; $2(2.5 \%)$ had oral candidiasis and $1(1.25 \%)$ had vaginal candidiasis.

Of 26 patients with TB, 24(92\%) were on ATT treatment. Of 80 seropositives in the study group, $28(35 \%)$ had $\mathrm{CD}_{4}$ counts in each categories of 401-800 and $>800.18(22.5 \%)$ had $\mathrm{CD}_{4}$ counts of 201-400. only $6\left(7.5 \%\right.$ had $\mathrm{CD}_{4}$ counts of $<200$.

The mean values of $\mathrm{CD}_{4}$ counts in the categories $<200,201-400,401-800,>800$ were $86.3,289.8,599.7$, 1654 respectively.

Among 80 seropositive individuals, 9(11.25\%) had WBC counts less than 4200 and $71(88.75 \%)$ had counts more than 4200. 8 of 9 individuals with WBC counts less than 4200 had $\mathrm{CD}_{4}$ less than 800 , ( $\left.\mathrm{p}=0.001\right)$. Of 80 individuals, $\mathrm{Hb}$ levels were available for $79.13(16.5 \%)$ had $\mathrm{Hb}$ levels less than $10 \mathrm{mg} \% .12$ of 13 individuals with $\mathrm{Hb}$ less than $10 \mathrm{mg} \%$ had $\mathrm{CD}_{4}$ counts less than $800,(\mathrm{p}=0.001)$. The majority in the study group, 41(51.3\%), had complaints of weight loss and $22(27.5 \%)$ had suffered diarrhoea at the time of sampling. All 22(100\%) with diarrhoea had presented with weight loss. All 22 had mean $\mathrm{CD}_{4}$ counts, mean WBC counts and mean $\mathrm{Hb}$ of $861.3,7800$ and 12.4 respectively. Of the 22 individuals with diarrhoea, $18(81.8 \%)$ had acute diarrhoea and 4(18.2\%) had chronic diarrhoea.

Among these 22, 20(90.1\%) yielded any of the opportunistic or non- opportunistic enteric pathogens on examination and only 2 cases with acute diarrhoea did not yield any pathogens on examination. $4(100 \%)$ cases with chronic diarrhoea had loose stools and all yielded opportunistic enteric protozoan parasites on examination. Of the 22 diarrhoeic stools of HIV cases, $13(59.1 \%)$ yielded parasites on wet mount examination; 1 of $13(7.7 \%)$ had opportunistic pathogen. None of the diarrhoeic stool samples of controls had opportunistic pathogens.

Of the 22 diarrhoeic stools of HIV cases, 7(31.8\%) yielded parasites on Kinyoun's staining; all $7(100 \%)$ 
were opportunistic protozoan pathogens. None of the diarrhoeic stool samples of controls had opportunistic pathogens.

The protozoan parasites recovered were Cryptosporidium sp. [Fig. 1], Microsporidium sp. [Fig. 2]; opportunistic helminthic parasite recovered was Strongyloides sp.

The helminthic parasites recovered were all non opportunistic pathogens. They were Entamoeba histolytica/dispar, Giardia sp., Ascaris sp., Ankylostoma sp.

Categorised by $\mathrm{CD}_{4}$ counts and diarrhoea symptom, the prevalence of intestinal parasitic infection as shown in table reveals $90 \%$ cases with diarrhoea had intestinal parasitic infection. The same was around $30 \%$ in non-diarrhoeics.

The prevalence of intestinal parasites among the HIV infected patients is as follows: Cryptosporidium

sp. and Microsporidium sp., each were found in $6.3 \%$ of HIV seropositive cases. Strongyloides was seen in $2.5 \%$ of 80 seropositive cases. The mean $\mathrm{CD}_{4}$ counts in cases with parasites are listed as follows: Cryptosporidium sp.-133.2 cells, Microsporidium sp.896 cells, Strongyloides stercoralis-228.5, Entamoeba histolytica/dispar-522 cells, Giardia sp.- 884 cells. Cryptosporidium sp. and Strongyloides stercoralis are found more in HIV seropositive cases with $\mathrm{CD}_{4}$ counts less than 200 cells; $(\mathrm{p}=0.002, \mathrm{OR}=36,95 \% \mathrm{CI}=4-56)$ for Cryptosporidium sp. and ( $\mathrm{OR}=14.6,95 \% \mathrm{CI}=1.5$ 39.6) for Strongyloides stercoralis.

There is a significant correlation between $\mathrm{CD}_{4}$ counts $<200$ and diarrhoea presentation, $(\mathrm{p}=0.005)$; increased risk, OR=16.76(95\% CI 1.63-106.59), [Table 1].

Table 1: Original: Distribution of diarrhoea cases among different $\mathrm{CD}_{4}$ cell counts

\begin{tabular}{|l|c|c|c|c|}
\hline $\begin{array}{c}\text { CD4 } \\
\text { count }\end{array}$ & $\begin{array}{c}\text { Total } \\
\text { examined }\end{array}$ & $\begin{array}{c}\text { Diarrhoea } \\
\text { present }\end{array}$ & $\begin{array}{c}\text { Crude odd's } \\
\text { ratio } \\
\text { (CI 95\%) }\end{array}$ & p-value \\
\hline$<200$ & 6 & 5 & $16.76(2.09-6.2)$ & 0.005 \\
\hline $201-400$ & 18 & 5 & $1.28(0.51-2.83)$ & $>0.05$ \\
\hline $401-800$ & 28 & 8 & $1.08(0.5-2.2)$ & $>0.05$ \\
\hline$>800$ & 28 & 4 & $0.3(0.15-1.1)$ & $>0.05$ \\
\hline Total & 80 & 22 & & \\
\hline
\end{tabular}

There seems to be an increased recovery of intestinal parasites from acute diarrhoea cases $(\mathrm{p}=0.01)$. This could be since, the present episode of diarrhoea could be an episodic occurrence of chronic diarrhoea, [Table 2].

Table 2: Original: Diarrhoea status among study group stratified by HIV status and parasitic infection

\begin{tabular}{|l|c|c|c|c|c|c|c|}
\hline \multirow{2}{*}{$\begin{array}{c}\text { Type of } \\
\text { diarrhoea }\end{array}$} & \multicolumn{2}{|c|}{ HIV Positive } & \multicolumn{2}{c|}{ HIV Negative } & \multicolumn{2}{c|}{ Total } & \multirow{2}{*}{ p-value } \\
\cline { 2 - 7 } & POrasite & $\begin{array}{c}\text { Parasite } \\
\text { Neg }\end{array}$ & $\begin{array}{c}\text { Parasite } \\
\text { POS }\end{array}$ & $\begin{array}{c}\text { Parasite } \\
\text { Neg }\end{array}$ & $\begin{array}{c}\text { HIV } \\
\text { POS }\end{array}$ & $\begin{array}{c}\text { HIV } \\
\text { NEG }\end{array}$ & \\
\hline Acute & 16 & 2 & 4 & 6 & 18 & 10 & 0.011 \\
\hline Chronic & 4 & 0 & 0 & 0 & 4 & 0 & NA \\
\hline No Diar & 16 & 42 & 2 & 8 & 58 & 10 & $>0.05$ \\
\hline Total & 36 & 44 & 6 & 14 & 80 & 20 & $>0.05$ \\
\hline
\end{tabular}

All the parasites were recovered in both the samples of a single case. Except Entamoeba histolytica/dispar, all the parasites were recovered after formol-ether concentration of stool samples. The children $<17$ years old are all inmates of the Shanthi Nivas school hostel premises. They are periodically dewormed and hence recovery of helminths is poor in this study group.

Microsporidia is been recovered from stool samples of HIV seropositive cases with $\mathrm{CD}_{4}$ counts $<800$, but not particularly in $\mathrm{CD}_{4}$ counts $<200$. This could be because we have taken cell counts done in the recent period and not done at the same time of sampling.

The area of sampling of cases and controls are different. No protozoan parasites have been recovered from controls. The recovery from cases should be truly pathogenic since only 6 of 80 cases have coccidian parasites isolated in them. It could be presumed that there is no water source contamination with protozoan parasites.

\section{Discussion}

Gastrointestinal involvement in HIV/AIDS is almost universal. Diarrhoea can be a presenting manifestation or a life threatening complication in HIV positive patients inducing weight loss and cachexia. Diarrhoea occurs in $90 \%$ of HIV infected population in developing countries. Infectious causes of diarrhoea have been found in $30-80 \%$ of patients and the etiology for such diarrhoea could be either parasitic, bacterial, fungal, enteric viruses or HIV itself. Recently diarrhoea 
without established etiology is prevalent in significant percentage of HIV infected population.

In the present study, a total of 80 HIV seropositive patients were investigated for opportunistic or nonopportunistic intestinal parasites. Symptomatic association of the parasites to diarrhoea presentation is discussed. Parasitic prevalence is correlated to the immune status of the HIV seropositive individuals. Majority of the patients (28\%) belonged to each of the age groups, 11-20 and 31-40 years. This indicates that the study group has majority, children with congenitally acquired HIV infection from parents and sexually active adults acquired HIV infection from their infected partners. Similar age related data has been provided by a study conducted by Beena uppal et al in Delhi. ${ }^{8}$

There were $58.7 \%$ of males in comparison to $41.3 \%$ of females with male to female ratio being 1.3:1. This male preponderance in the present study also coincides with the findings of a study done by Kava Mohandas et al in North India. ${ }^{9}$

The presentations like diarrhoea, fever, weight loss in the study group is consistent with the clinical presentations documented in other studies. ${ }^{10}$ Mycobacterium tuberculosis was the commonest opportunistic pathogen reported in few studies from India. Pulmonary Tuberculosis was observed in 35\% of the study group by Singh A et al in a study from Manipal. ${ }^{11}$

In the present study $27.5 \%$ of patients suffered with diarrhoea (acute and chronic). Prevalence of diarrhoeal illness in HIV seropositive patients ranging from $28 \%$ to $47 \%$ has also been documented by other authors., ${ }^{4,12}$

Enteric pathogens were detected from stool in $90 \%$ of diarrhoeal patients compared to $30 \%$ of those without diarrhoea. $31.3 \%$ of HIV seropositive patients in the present study suffered from protozoal infestations. A prevalence of rate of $35 \%$ has also been observed in Pune. ${ }^{13}$

In the present study detection of Cryptosporidium (6.3\%) was consistent with other studies. ${ }^{14}$

Other enteric protozoal pathogens causing infections in HIV seropositive patients like Cyclospora cayetanensis and Isospora belli were not seen in the present study because of possible geographical variations in the prevalence of these pathogens in HIV infected patients.

A definitive diagnosis of most of the parasitic infections depends on the demonstration of trophozoites, ova and cyst. This is traditionally done by stool microscopic examination, which is the gold standard in the diagnosis of intestinal parasitic infections.

A comparative study done by Kehl et $\mathrm{al}^{15}$ by comparing four staining methods for detection of Cryptosporidium oocysts in faecal specimens from the field, and in another study done by MacPherson and McQueen ${ }^{16}$ in multiattribute evaluation of six diagnostic methods, ranks the Modified Ziehl Neelsen stain as the best method for staining Cryptosporidium oocysts. This staining method used in our study showed a clear distinct morphological appearance and bright pink appearance of oocyst which helps to spot the parasite in a blue background.

The most striking finding of our study was the high prevalence of Enterocytozoon bieneusi infection. Microscopic stool examination by Kinyoun's staining method demonstrated that $6.3 \%$ of HIV infected individuals were infected with this parasite. Kumar SS et $\mathrm{al}^{17}$ demonstrated $1.6 \%$ prevalence of microsporidia among HIV infected cases with diarrhoea in Chennai by this staining while it was $15.9 \%$ in a study by Saigal et al. ${ }^{18}$ A study in North India by Kava Mohandas et al using trichrome staining showed $2.5 \%$ prevalence rate; ${ }^{9}$ study in Thailand using trichrome staining demonstrated that the prevalence of E. bieneusi infection in HIV-infected patients with diarrhea was $1.67 \% .^{19}$

Normally microsporidia is demonstrated effectively by trichrome staining using chromotrope $2 \mathrm{R}$ or calcoflour white staining based on flourescent microscope. It is ranked the best in a recent study done at Varanasi and by Didier et al. ${ }^{20}$

Strongyloides stercoralis was found in $2.5 \%$ of HIV infected cases. Prevalence of $1.67 \%$ among HIV seropositive cases with diarrhoea was seen in Indian studies. ${ }^{21}$ Strongy loides infection in its endemic tropical countries is mostly asymptomatic but hyperinfection with it, in HIV infected population, is an AIDS defining diagnosis (CDC,1986), accounts for a high mortality rate.

The $10 \%$ occurrence of Giardia lamblia in this study and that of E.histolytica/dispar is $8.8 \%$, tallies with study by Kava Mohandas et al. ${ }^{9}$

Neither they were strongly associated to chronic diarrhoea symptom nor to the HIV status. Cellular immune responses play major role in defence against Entamoeba but their etiological role in parasitic diarrhoea in HIV seropositive cases is not yet established. Studies in America reveal that they are predominant in HIV seropositive male homosexuals. Host defences against Giardia seems to be humoral and secretory immunoglobulins. The lack in adequate mucosal immune responses has led to increased rate of Giardia infection in HIV seropositive cases.

Our study has several limitations. Because only a single stool concentration technique was used, the prevalence of infection may have been underestimated. Furthermore, the role of bacterial and viral pathogens was not investigated. Finally, CD4 cell counts were not determined at the time of stool sampling, instead, CD4 counts measured at the nearest time period to sampling was taken from records and therefore the true level of immunosuppression is unknown.

The areas of sampling of cases and controls were entirely different. Hence, variations in the prevalence of parasites are attributable to geographic conditions. 
Non-opportunistic pathogens like Ascaris sp., Ankylostoma sp., Hymenolepis nana are prevalent in both cases and control groups but the suppressed immune status in HIV seropositive group explains the marginally increased percentage of parasite prevalence in them.

\section{Conclusion}

Cryptosporidiosis is the commonest infection, which plays a major role in the epidemiology of opportunistic infections among the enteric parasites in HIV infected individuals.

In India particularly south India, Cryptosporidium remains a predominant protozoan parasite in HIV seropositive patients. This is evident by the studies done by different authors across south India.

The present study also shows that Cryptosporidium is the predominant enteric protozoan parasite.

In our study the rarest and frequently not recognised parasite in India, Microsporidia, is detected in good number of cases.

Hence it is necessary that HIV/AIDS patients presenting with diarrhoea should be screened for enteric protozoan parasites with the most accurate staining methods.

Although our study was confined to a limited number of sample size, it confirms the findings that HIV seropositive individuals are more prone to infections with enteric protozoan parasites belonging to both opportunistic and non-opportunistic pathogens.

A knowledge and diagnosis of the opportunistic infections in HIV patients would help clinicians and health planners to tackle the AIDS epidemic in a more effective manner.

Funding: No funding sources.

Conflict of interest: None declared.

\section{References}

1. UNAIDS (2017) 'Fact Sheet 2017'

2. UNAIDS (2017) Data Book

3. Jha AK, Uppal B, Chadha S, Bhalla P, Ghosh R, Aggarwal P, et al. Clinical and microbiological profile of HIV/AIDS cases with diarrhea in North India. $J$ Pathol 2012;2012:7.

4. Tiwari BR, Ghimire P, Malla S, Sharma B, Karki S. Intestinal parasitic infection among the HIV-infected patients in Nepal. J Infect Dev Ctries 2013;7(7):550-55.

5. Oldfield C Edward. Evaluation of Chronic diarrhoea in patients with Human Immunodeficiency virus infection. Reviews in Gastroenterological disorders 2002 ; 2(4) : 176-188.
6. Edward A et al. Diarrhoeal Diseases Associated with HIV infection. Gastroenterol Clin N Am June 1997;26(2):259-90.

7. Talib SH, Jeet Singh. A study on Opportunistic enteric parasites in 80 HIV seropositive patients. IJPM 1998 ; 41 (1) : 31-37.

8. Beena Uppal, Bineeta Kashyap, Preena Bhalla. Enteric pathogens in HIV/ AIDS from a tertiary care hospital. Indian J Community Med 2009:34(3):237-42.

9. Kava Mohandas, Rakesh Segal, Archana Sud and Nancy Malla. Prevalence of Intestinal Parasitic Pathogens in HIV-Seropositive Individuals in Northern India. Jpn J Infect Dis 2002:55:83-4.

10. Feasey NA, Healey P, Gordon MA. Review article: the aetiology, investigation and management of diarrhea in the HIV-positive patient. Aliment Pharmacol Ther 2011:34:587-603.

11. Singh A, Bairy I, Shivananda PG. Spectrum of opportunistic infections in AIDS cases. Indian J Med Sci 2003;57:16-21.

12. S. Gupta, S. Narang, V. Nunavath, and S. Singh, Chronic diarrhoea in HIV patients: prevalence of coccidian parasites. Indian J Med Microbiol 2008:26(2):172-75.

13. S.V Kulkari, R.Karion,S. S. Sane, P.S. Padmavar. Opportunistic parasitic intestinal infections in HIV/AIDS Patient presenting with diarrhoea by the level of immunesuppression. Indian J Med Res 2009:63-6.

14. NARI. Study on Opportunistic enteric parasites in HIV seropositive adult patients hospitalized for diarrhea. 2002.

15. Kehl KSC, Cicirello H, Havens PL: Comparison of Four Different Methods for Detection of Cryptosporidium Species. J Clin Microbiol 1995:33:416-18.

16. MacPherson DW, McQueen R: Cryptosporidiosis: Multi attribute Evaluation of Six Diagnostic Methods. J Clin Microbiol 1993:31:198-202.

17. Kumar SS, Ananthan S, Lakshmi P. Intestinal parasitic infection in HIV infected patients with diarrhoea in Chennai. Indian J Med Microbiol 2002;20:88-91.

18. Saigal K, Sharma A, Sehgal R, Sharma P, Malla N, Khurana S. Intestinal microsporidiosis in India: a two year study. Parasitol Int 2013;62(1):53-6.

19. Viroj Wiwanitkit. Intestinal parasitic infections in Thai HIV-infected patients with different immunity status. BMC Gastroenterol 2001;1:322.

20. Didier ES, Orenstein JM, Aldras A, Bertucci D, Rogers LB, Janney FA: Comparison of Three Staining Methods for Detecting Microsporidia in Fluids. J Clin Microbiol 1995;33:3138-45.

21. K.N. Prasad, V.L. Nag, T.N. Dhole, and A. Ayyagari. Identification of Enteric Pathogens in HIV-positive Patients with Diarrhoea in Northern India. J Health Popul Nutr 2000;18(1):23-6.

How to cite this article: G. Hemalatha, T. Mangaiyarkarasi, A. Malini, Banoo S., K. Sethmadhavan. A study of intestinal parasitic infections in HIV infected individuals in Puducherry. Int $\mathrm{J} \mathrm{Med}$ Microbiol Trop Dis 2018;4(4):266-71. 